I N F E C T I O U S C H A N G E 

K A THER I N A M A S N

\section{Infectious Change}

Reinventing Chinese Public Health

After an Epidemic 
Stanford University Press

Stanford, California

(C) 2016 by the Board of Trustees of the Leland Stanford Junior University. All rights reserved.

No part of this book may be reproduced or transmitted in any form or by any means, electronic or mechanical, including photocopying and recording, or in any information storage or retrieval system without the prior written permission of Stanford University Press.

Printed in the United States of America on acid-free, archival-quality paper

Library of Congress Cataloging-in-Publication Data

Names: Mason, Katherine A. (Katherine Anne), author.

Title: Infectious change : reinventing Chinese public health after an epidemic / Katherine A. Mason.

Description: Stanford, California : Stanford University Press, 20I6. I Includes bibliographical references and index.

Identifiers: LCCN 2015050118 I ISBN 9780804794435 (cloth : alk. paper) | ISBN 978080479892 I (pbk. : alk. paper) I ISBN $978080479895^{2}$ (ebook)

Subjects: LCSH: Public health-China, Southeast. I Public health administration-China, Southeast. I SARS (Disease)—China, Southeast.

Classification: LCC RA395.C6 M37 2016 I DDC 362.1095 I-dc23

$\mathrm{LC}$ record available at http://lccn.loc.gov/2015050I 18

Typeset by Thompson Type in 9.75/13.5 Janson 
For my family: past, present, and future 
